\title{
Le droit et la sexualité
}

\author{
A. WEIL-RAYNAL \\ Avocat à la Cour, 61 Bd St Michel, 75005 Paris
}

La Loi s'est toujours crue investie d'un droit de regard sur la sexualité des individus.

Le droit canon, droit religieux sur lequel a longtemps reposé le droit français, réprouvait, toutes causes confondues, la fornication, le stupre, le concubinage, l'inceste, le rapt, la sodomie, le viol, la bestialité, la bigamie et l'adultère.

Les mœurs ont évolué. La France a réformé son droit, mais la Loi a continué de régir fermement le sexe dans l'ensemble de ses manifestations.

Bien sûr, on ne brûle plus aussi facilement que sous l'Ancien Régime (ex: Gilles de Rais avouant le viol, la torture et l'assassinat de nombreux petits garçons en 1442), mais les prétoires retentissent encore régulièrement des anodines provocations entre époux, comme des perversions les plus ignominieuses.

Parmi les quelques dizaines de milliers de procès annuels où les mœurs sont en cause, tous ne relèvent pas du domaine pénal: la sexualité a également d'autres incidences juridiques, tant sur le mariage que sur la paternité, le changement de sexe, la procréation médicalement assistée ou bien encore sur les libertés administratives de projeter tel ou tel film ou d'aller et venir pour les péripatétitiennes.

Le droit s'immisce dans tous les domaines, y compris les plus intimes: le législateur ne pouvait se désintéresser du sexe et de la sexualité, y compris dans les branches les plus insoupçonnables, jusqu'au droit fiscal qui s'ingénie le plus à traquer l'activité sexuelle.
Le contrôle fiscal lui-même opère déjà des ravages: «Quand un commerçant vient me consulter pour impuissance, confie un sexologue connu, je lui demande tout de suite s'il a reçu la visite des polyvalents. Je me trompe rarement".

$\mathrm{Si}$, a priori, il n'y a aucun rapport entre la sexualité et l'impôt, les pouvoirs publics disposent cependant là d'un instrument susceptible d'influer sur le comportement sexuel des contribuables en les incitant par exemple, au gré de la législation en vigueur, à rendre féconds ou stériles leurs ébats, dans des draps légaux ou non.

Un récent rapport de l'INSEE n'attribue-t-il pas la forte hausse des mariages observée en 1996 , non pas à un retour en force des valeurs morales, mais au vote de la loi de finances rétablissant une égalité de statuts entre les couples mariés et les concubins?

Le régime fiscal des revenus de la prostituée ou de son souteneur relève des bénéfices non commerciaux; quant à ceux des organisateurs de réseaux ou des exploitants de maisons closes, l'administration les range dans la catégorie des bénéfices industriels et commerciaux et ils sont à ce titre passibles de la T.V.A.

Enfin, le droit social lui-même est concerné par le sexe, à en croire cette décision du Conseil des Prud'hommes d'Autun considérant que l'excuse de «masturbation» - qualifiée de légitime satisfaction - n'était pas un motif de licenciement (24 mars 1992).

Le législateur n'a jamais devancé l'évolution 
des mœurs : avec un ou plusieurs trains de retard, il n'a fait que s'adapter à des mutations dont il serait réducteur de penser qu'elles n'ont progressé que dans le sens d'un plus grand laxisme, ce que pourrait laisser penser la libéralisation générale des mentalités.

Bien sûr on ne brûle ni même ne décapite plus les criminels, même si certains partisans $d u$ rétablissement de la peine de mort ne l'appellent plus de leurs vœux que pour les seuls tortionnaires d'enfants, majoritairement coupables de sévices sexuels.

L'adultère n'est plus un délit depuis 1975, mais il est resté une cause de divorce presque assurée.

Mais les viols systématiquement correctionnalisés pendant de longues années sont redevenus passibles, sous la pression des mouvements féministes, des Cours d'Assises.

Plus encore, la qualification de viol est à présent retenue pour la fellation (arrêt de la Cour de Cassation 22 février 1984) après avoir été longtemps considérée comme un simple attentat à la pudeur.

Enfin, la notion de viol entre époux a également été consacrée par la Cour de Cassation dans une décision (11 juin 1992) aux termes de laquelle " la présomption de consentement des époux aux actes sexuels accomplis dans l'intimité de la vie privée conjugale ne vaut que jusqu'à preuve contraire", alors que le vol entre époux n'existe toujours pas.

Et pourtant la société n'a-t-elle pas de tous temps protégé aussi jalousement la propriété que l'intimité?

Ce n'est là qu'un seul des aspects des rapports ambigus qu'entretiennent le sexe et la loi.

Plus récemment encore, notre Nouveau Code Pénal a donné le jour au délit de harcèlement sexuel.

Et les médecins?

Le geste médical, en matière sexuelle, peut lui aussi comporter des conséquences juridiques sans parler de celui de l'accoucheur source par excellence de vie et donc de droit,

-c'est celui du chirurgien, capable de modifier le sexe, tant dans son apparence que jusque dans l'état civil.

En effet, un arrêt de la Cour de Cassation du 11 septembre 1992 admet que lorsqu'à la suite d'un traitement médico-chirurgical subi à des fins thérapeutiques, une personne présentant le syndrome de transsexualisme ne possède plus tous les caractères de son sexe d'origine et a pris une apparence physique se rapprochant de l'autre sexe, auquel correspond son comportement sexuel; le principe du respect dû à sa vie privée justifie que son état civil indique désormais le sexe dont elle a l'apparence.

-c'est aussi le signalement par le médecin des agressions sexuelles qu'il constate dans l'exercice de sa profession: le législateur dans ce cas le délie de l'obligation du respect du secret professionnel, mais impose en corollaire le respect d'une autre condition : le consentement de la victime.

-c'est encore celui du prescripteur d'anti-androgènes, capable de freiner à l'aide d'un médicament l'activité sexuelle d'un individu lorsque cette dernière sort de la légalité.

Dans ce cas, il sera relevé l'intervention du Comité National d'Ethique, qui a émis un avis sur ce qui a pu être considéré soit comme une castration chimique, soit comme la solution idéale à l'inefficacité conjuguée de la Justice et de la Médecine : la prescription de substances antiandrogéniques à des détenus condamnés pour des infractions à caractère sexuel.

Le docteur Bernard Cordier, psychiatre et expert près la cour d'Appel, se fait l'écho, à propos de cette prescription à des patients en liberté, la plupart pédophiles, qui ne parvenaient pas à contrôler leurs pulsions sexuelles, d'une part de la méfiance que suscitent en général les substances chimiques susceptibles de modifier le comportement d'un être humain, et d'autre part du vécu de ce traitement par certains de ses patients comme une libération vis-à-vis d'une sexualité contraignante dont ils se sentaient prisonniers et qui créaient chez eux un sentiment d'aliénation.

-enfin, c'est le geste qui vous permet à vous, urologues, andrologues, et qui permettra demain à tous les généralistes, de redonner la vigueur aux membres virils défaillants. 
Faudra-t-il attendre un fait divers retentissant pour que le Comité National d'Ethique s'intéresse à ce geste, à savoir la capacité de restituer à l'homme impuissant les forces de Priape $?$

La responsabilité du prescripteur pourra-t-elle être engagée si son patient revigoré use et abuse de ses forces nouvelles pour violenter un enfant, alors que le médecin connaissait ses perversions, ou qu'il n'a pas procédé à des investigations suffisamment approfondies pour acquérir la conviction qu'il était exempt de tels fantasmes?

Ne pourra-t-il pas dans ces conditions être poursuivi pour complicité par fourniture de moyens, ou plus simplement sur le fondement de sa responsabilité civile?

«Les médecins écrivait Voltaire, sont des gens qui introduisent dans le corps humain qu'ils connaissent mal, des substances qu'ils connaissent encore moins bien.»

En conclusion, et d'un mot, la considération du sexe par le droit concerne au plus profond d'elle-même l'humanité, son devenir et le fondement du droit. 